\title{
Analyses of chlorogenic acids and related cinnamic acid derivatives from Nicotiana tabacum tissues with the aid of UPLC-QTOF-MS/MS based on the in-source collision-induced dissociation method
}

\author{
Efficient N Ncube ${ }^{1}$, Msizi I Mhlongo ${ }^{1}$, Lizelle A Piater ${ }^{1}$, Paul A Steenkamp ${ }^{1,2}$, lan A Dubery ${ }^{1}$ and Ntakadzeni E Madala ${ }^{1 *}$
}

\begin{abstract}
Background: Chlorogenic acids (CGAs) are a class of phytochemicals that are formed as esters between different derivatives of cinnamic acid and quinic acid molecules. In plants, accumulation of these compounds has been linked to several physiological responses against various stress factors; however, biochemical synthesis differs from one plant to another. Although structurally simple, the analysis of CGA molecules with modern analytical platforms poses an analytical challenge. The objective of the study was to perform a comparison of the CGA profiles and related derivatives from differentiated tobacco leaf tissues and undifferentiated cell suspension cultures.

Results: Using an UHPLC-Q-TOF-MS/MS fingerprinting method based on the in-source collision induced dissociation (ISCID) approach, a total of 19 different metabolites with a cinnamic acid core moiety were identified. These metabolites were either present in both leaf tissue and cell suspension samples or in only one of the two plant systems. Profile differences point to underlying biochemical similarities or differences thereof.

Conclusion: Using this method, the regio- and geometric-isomer profiles of chlorogenic acids of the two tissue types of Nicotiana tabacum were achieved. The method was also shown to be applicable for the detection of other related molecules containing a cinnamic acid core.
\end{abstract}

Keywords: Nicotiana tabacum, Cell suspensions, Chlorogenic acid, Cinnamic acid, ISCID, Leaf tissue, UPLC-qTOF-MS/MS

\section{Background}

Chlorogenic acids (CGAs) are the family of ester phytochemicals formed between cinnamic acid derivatives and quinic acids. These compounds are present in almost all plants and contribute a significant fraction of the total dietary intake of phenols in the daily human diet. Moreover, they possess some notable bio-medical or pharmacological properties [1-3]. CGAs are phenolic compounds produced through the shikimate- and phenylpropanoid pathways [4,5], and have been identified in responses against both biotic and abiotic stressors [6]. The most common naturally-occurring cinnamic acid derivatives that have been reported to be utilized during the

\footnotetext{
* Correspondence: emadala@uj.ac.za

${ }^{1}$ Department of Biochemistry, University of Johannesburg, P.O. Box 524,

Auckland Park 2006, South Africa

Full list of author information is available at the end of the article
}

biosynthesis of these molecules are $p$-coumaric -, caffeicand ferulic acid which give rise to $p$-coumaroylquinic acid ( $p$ CoQA), caffeoylquinic acid (CQA) and feruloylquinic acid (FQA), respectively $[1,2]$. Other acids such as sinapic acids are, however, rarely found.

Naturally, plants are known to synthesize the transisomers over the cis-isomers of CGA compounds. The latter has been reported to be formed in tissue or extracts previously exposed to UV light, mechanical processing of coffee and electric field during MS data acquisition [7]. However, our latest study on tobacco cells treated with different chemical and biological priming inducers shows that the cis-isomer of 5-CQA was induced or up-regulated from a pre-existing pool when compared to non-treated cells. These results suggest the existence of possible enzymatic machinery responsible for the production of the cis-isomers in plants [8]. From 
an analytical perspective, CGA molecules offer a challenge owing to the structural similarities and complexity of these compounds. As such, scientists still spend a great deal of time on developing appropriate methodologies, even though reliable methods for the analysis have been developed in the past $[8,9]$. Most of the approaches for the analysis of these compounds are, however, achieved by the use of ion trap MS-based platforms [1,2,10-12]. Due to the limited availability of such instruments, emphasis is being placed on the development of alternative, equivalent methods to overcome these challenges. The development of the ion trap hierarchical approach has significantly contributed to the detailed analyses of CGA molecules [10-14]. Apart from the structural hierarchy, there exists regio- and geometric-isomerism which offers yet another dimension of complexity to the analyses of these molecules. However, the use of such ion trap-MS methods has also enabled the discrimination between regio- but not geometric-isomers of CGAs. As such, chromatographic methods have also been optimized for proper annotation. The order of elution of the mono-acylated CGA seems to remain constant on a reverse-phase column [12]. Using this knowledge, other methods based on Q-TOF-MS have been developed [7], albeit in most cases with several difficulties which ultimately affect the accuracy of the annotated metabolites.

To circumvent this problem, we have recently developed a Q-TOF-MS fingerprinting method based on the in-source collision induced dissociation (ISCID) approach for the analysis of chlorogenic acid derivatives [8]. This method has proved to generate very stable and reproducible results compared to previously published data. To substantiate our method, the current study profiles the CGA content of tobacco plant systems (leaf tissue vs. cultured cells) which have been reported to be a good source of a variety of bioactive constituents including CGAs [15].

For plant-related studies, mass production of secondary metabolites can be achieved by using plant cell suspensions. Cultured cells do not only provide a costeffective alternative as they are also environmentally friendly. The main advantage of using this system is that it can be easily manipulated for biotechnological purposes $[16,17]$. However, it should be noted that cells in suspension culture differ significantly from mature organ tissue such as leaves in that cells grow rapidly and divide, do not contain any traces of chlorophyll and multiply in an aqueous environment containing stimulatory phytohormones [18]. Thus, the response to stress factors encountered by intact plant leaves and cell suspensions may vary. The evident biochemical differences between cell suspensions and leaf tissues have also been attributed to the different environmental conditions to which they are exposed to [19]. Furthermore, it has been reported that the biosynthesis of CGAs is highly dependent on the developmental stage of the tissues [20]. Cell type-associated localization of CGAs during maturation (i.e. tissue development) has also been reported [21].

As such, the aim of the current study was to comprehensively profile the CGA content of both tobacco leaf tissue and cell suspensions. The results of the current study are also expected to contribute to the identification of any underlying biochemical differences with regard to CGA biosynthesis between the two systems.

\section{Experimental \\ Plant material}

Nicotiana tabacum cv. Samsun cell cultures were grown in a Murashige and Skoog (MS) medium containing 0.25 $\mathrm{mg} / \mathrm{L}$ 2,4-dichlorophenoxyacetic acid and $0.25 \mathrm{mg} / \mathrm{L}$ kinetin ( $\mathrm{pH}$ 5.8) [22] at room temperature on a shaker at $120 \mathrm{rpm}$ with a light/dark cycle of $12 \mathrm{~h} / 12 \mathrm{~h}$, and low light intensity of $30 \mu \mathrm{mol} / \mathrm{m}^{2} / \mathrm{s}$. Tobacco plants were grown in composted soil under greenhouse conditions: temperature min $10^{\circ} \mathrm{C}$ and $\max 22^{\circ} \mathrm{C}$, light/dark cycle of $12 \mathrm{~h} / 12 \mathrm{~h}$, and light intensity of $60 \mu \mathrm{mol} / \mathrm{m}^{2} / \mathrm{s}$.

\section{Extraction of metabolites}

The cells were harvested by filtration on $55 \mathrm{~mm}$ filter paper circles using a vacuum filtration system (Millipore, Billerica, MA, USA) and washed with $20 \mathrm{~mL}$ MS medium without vitamins while tobacco leaves were ground with a mortar and pestle in liquid nitrogen. Two grams $(2 \mathrm{~g})$ of each sample was weighed and homogenized in $20 \mathrm{~mL}(1: 10 \mathrm{~m} / \mathrm{v}) 80 \%$ methanol using a probe sonicator (Bandelin Sonopuls, Germany) set at 55\% power for $15 \mathrm{sec}$ with 4 cycles. The crude extract was centrifuged at $4100 \times \mathrm{g}$ for $15 \mathrm{~min}$ at room temperature. The supernatant was evaporated to approximately $1 \mathrm{~mL}$ using a rotary evaporator set at $55^{\circ} \mathrm{C}$ before being dried to completeness in a heating block set at $55^{\circ} \mathrm{C}$ overnight. The dried residues were reconstituted in $300 \mu \mathrm{L} \mathrm{50 \%} \mathrm{(v/}$ v) UHPLC-grade methanol in milli-Q water, and filtered through a $0.22 \mu \mathrm{m}$ nylon filter into glass vials fitted with $500 \mu \mathrm{L}$ inserts. The filtered extracts were stored at $-20^{\circ} \mathrm{C}$ until analysis. For reproducibility of the results, the experiments consisted of 5 independent biological repeats and each extract was analyzed in duplicate ( 2 technical repeats).

\section{Ultra-high performance liquid chromatography (UPLC)}

The extracts were chromatographically analyzed on a UHPLC high-definition quadrupole time-of-flight MS instrument (UPLC-qTOF SYNAPT G1 HDMS system, Waters Corporation, Manchester, UK) fitted with an Acquity HSS T3 column $(1.7 \mu \mathrm{m}, 2.1 \times 150 \mathrm{~mm}$; Waters Corporation). A binary solvent system consisting of eluent A: $0.1 \%$ formic acid in water and B: $0.1 \%$ formic acid in acetonitrile (Romil Chemistry, UK) was used. A $20 \mathrm{~min}$ 
gradient method at constant flow rate of $0.4 \mathrm{~mL} / \mathrm{min}$ was used for analyte separation, and the conditions were: 2\% B over $0.0-1.0 \mathrm{~min}, 2-3 \% \mathrm{~B}$ over $1.0-3 \mathrm{~min}, 3-8 \% \mathrm{~B}$ over 3-4 min, 8-12\% B over 4-12.00 min, 12 - 95\% B over 12-13 min and held constant at $95 \%$ B over $13-15$ min to wash the column and $95-5 \%$ B over $15-16 \mathrm{~min}$. Thereafter, the column was returned to initial conditions at $16 \mathrm{~min}$ and allowed to equilibrate for $4 \mathrm{~min}$. Chromatographic separation was monitored using a photodiode array (PDA) detector (Waters Corporation, UK) with a scanning range set between 200-500 nm, $1.2 \mathrm{~nm}$ bandwidth resolution and a sampling rate of 20 points/sec.

\section{Quadrupole time-of-flight mass spectrometry (Q-TOF-MS)}

Post-PDA detection, the metabolites were further detected with the aid of a SYNAPT G1 high definition mass spectrometer (Waters Corporation, UK) operating in negative ionization mode. The MS conditions were as follows: capillary voltage of $2.5 \mathrm{kV}$, sample cone voltage of $30 \mathrm{~V}$, microchannel plate (MCP) detector voltage of $1600 \mathrm{~V}$, source temperature of $120^{\circ} \mathrm{C}$, desolvation temperature of $450^{\circ} \mathrm{C}$, cone gas flow of $50 \mathrm{~L} / \mathrm{h}$, desolvation gas flow of $550 \mathrm{~L} / \mathrm{h}, \mathrm{m} / z$ range of $100-1000$, scan time of $0.2 \mathrm{sec}$, interscan delay of $0.02 \mathrm{sec}$, mode set as centroid, lockmass set as leucine enkephalin (554.2615 Da), lockmass flow rate of $0.1 \mathrm{~mL} / \mathrm{min}$, and mass accuracy window of $0.5 \mathrm{Da}$. High purity nitrogen was used as desolvation gas, cone - and collision gas.

\section{In-source collision-induced dissociation (ISCID)}

Tandem MS $\left(\mathrm{MS}^{2}\right)$ fragments of the chlorogenic acids were generated by an ISCID approach. For quinic acid bearing molecules, the approach was optimized for the detection of $m / z 191$ [quinic acid- $\mathrm{H}^{+}$] and a similar method was used for the other molecules herein. For CGA characterization the trap collision $(30 \mathrm{eV})$ and cone energy $(60 \mathrm{~V})$ was experimentally optimised until stable fragmentation was obtained characterized by the formation of $\mathrm{C} 1$ [caffeic acid-H], $\mathrm{C} 2$ [caffeic acid- $\mathrm{CO}_{2}$ ], Q1 [quinic acid-H] and Q2 [quinic acid- $\mathrm{H}_{2} \mathrm{O}$ ] ions (for CQA as an example).

\section{Results and discussion}

Due to the lack of authentic standards and low concentration of metabolites, the current study only presents qualitative data. In most metabolite fingerprinting studies, metabolite identification is challenging and requires the use of authentic standards for definite metabolite identification. However, due to unavailability of most plants standards, we opted for cider and coffee as surrogate extracts for some of the metabolites identified herein [12]. It is also worth mentioning that the introduction of instruments such as Q-TOF-MS have contributed significantly in overcoming this problem because of its ability of measuring mass accuracy below 3 ppm [23]. For all the 19 metabolites detected (Figure 1, Table 1), the mass error was below $5 \mathrm{ppm}$. As previously mentioned, proper annotation of structurally similar isomers of cinnamic acid derivatives is a difficult task [9]. To avoid ambiguity during identification, using the IUPAC numbering system, we have considered all possible factors ranging from chromatographic separation to mass spectrometric behaviour as shown in previously published data $[10,12]$. In order to simplify identification, metabolites harboring common cinnamic acid moieties in their core structure were monitored using a single mass extracted ion chromatogram (XIC). As an example, four peaks with $m / z$ 353 , representative of three regio-isomers of CQA, were detected in extracts from both cells and leaves (Figure 2). This enabled proper identification of CGAs based on previously published data, taking into consideration all possible isomers both at positional and geometrical level [1,2,10-12,23]. It has been reported that CGA molecules fragment in a similar manner under well-optimized conditions. For instance, CQAs are known to result in the following fragment ions: Q1 [quinic acid-H], C1 [caffeic acid-H], Q2 [quinic acid- $\mathrm{H}_{2} \mathrm{O}$ ] and $\mathrm{C} 2$ [caffeic acid- $\mathrm{CO}_{2}$ ] [24]. By monitoring these ions, CGA and the related derivatives could be positively annotated as discussed below. All mass spectra shown in Figures 3, 4, 5, 6 and 7 were generated at a collision energy of $30 \mathrm{eV}$ and $60 \mathrm{eV}$ cone voltage as described under Experimental section.

\section{Characterization of $p$-coumaroylquinic acids}

$p$-Coumaroylquinic acid has a molecular weight $(\mathrm{Mr})$ of 338 and four peaks at $m / z 337$ were detected in leaf extracts whilst only three were detected in cells (XIC not shown). These ion peaks, according to the mass spectra (Figure 3), were identified as trans-4- $p$ CoQA (1), trans5-pCoQA (2), cis-5-pCoQA (3) and cis-4-pCoQA (4). The leaf samples were found to contain 1, 2, 3 and 4 while the cells were found to only contain 1, 2 and 4 . The absence of 3 (cis-5-pCoQA) in cells could not be entirely explained but is possibly due to the fact that some molecules are more prone to isomerization than others, a phenomenon which has been observed in other plants but without supporting explanation [8]. In addition, another important factor to note is that both trans- and cis-isomers have very similar fragmentation patterns [12]. In the current study the mass spectra of the trans isomers are shown throughout. The notable absence of 3$p$ CoQA in both leaf and cell extracts is an observation which represents a very interesting biochemical phenotype. This molecule has been found to accumulate in other plants such as legume forages and birch trees [25-27]. 


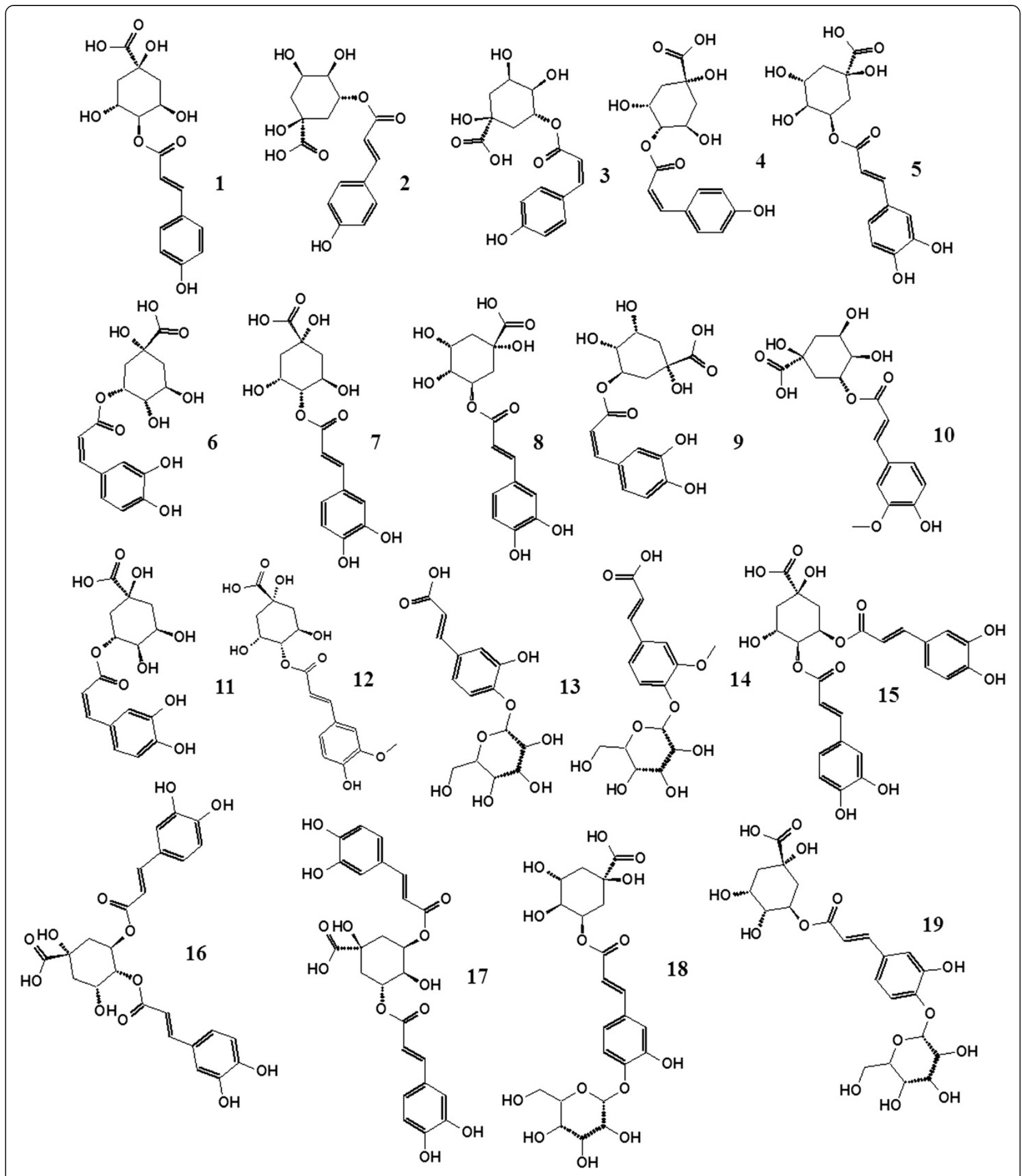

Figure 1 The structures of chlorogenic acids and related derivatives and CQA glycosides detected in tobacco leaves and cultured cells.

\section{Characterization of caffeoylquinic acids}

Although present at different relative intensities, all CQA regio-isomers $(\mathrm{Mr}=354)$ were identified in both tobacco leaves and cultured cells: trans- and cis-3CQA $(\mathbf{5}, \mathbf{6})$, trans-4-CQA (7) and trans- and cis-5-CQA $(\mathbf{8}, \mathbf{9})$
(Figure 4). Amongst the CGAs, the CQAs have the widest occurrence and are well researched $[21,28]$. Unlike $p$ CoQA, only 3- and 5-acylated CQA possessed both cis- and transisomers whilst only the trans-isomer of 4-CQA (7) was detected. All the mentioned CQA molecules were found in 
Table 1 Characterization of chlorogenic acids and related cinnamic acid derivatives detected in tobacco leaves and cells

\begin{tabular}{llllll}
\hline Compound name & Retention time (min) & Cells & Leaves & [M-H] $^{-}$ & Diagnostic $\mathbf{m} / \mathbf{z}$ ions \\
\hline cis-4-pCoQA (4) & 7.35 & $\checkmark$ & $\checkmark$ & 337.0933 & 173 \\
trans-4- pCoQA (1) & 7.96 & $\checkmark$ & $\checkmark$ & 337.0957 & 173 \\
trans-5-pCoQA (2) & 7.69 & $\checkmark$ & $\checkmark$ & 337.0886 & 191 \\
cis-5-pCoQA (3) & 10.54 & & 337.0949 & 191 \\
cis-3-CQA (6) & 4.55 & $\checkmark$ & $\checkmark$ & 353.0875 & $191,179,135$ \\
trans-3-CQA (5) & 4.61 & $\checkmark$ & $\checkmark$ & 353.0787 & $191,179,135$ \\
trans-4-CQA (7) & 6.13 & $\checkmark$ & $\checkmark$ & 353.0864 & $191,179,173,135$ \\
trans-5-CQA (8) & 5.81 & $\checkmark$ & $\checkmark$ & 353.0829 & 191,135 \\
cis-5-CQA (9) & 7.55 & $\checkmark$ & $\checkmark$ & 353.0809 & 191,135 \\
trans-4-FQA (12) & 6.17 & & $\checkmark$ & 367.0429 & 173 \\
trans-5-FQA (10) & 5.84 & $\checkmark$ & $\checkmark$ & 367.0847 & 191 \\
cis-5-FQA (11) & 9.41 & $\checkmark$ & $\checkmark$ & 341.0894 & 191 \\
Caffeoylglycoside (13) & 5.60 & $\checkmark$ & 355.1012 & 179 \\
Feruloylglycoside (14) & 7.09 & $\checkmark$ & 515.1509 & 193 \\
3,4-diCQA (15) & 5.21 & & $\checkmark$ & 515.1404 & $191,179,173,135$ \\
4,5-diCQA (16) & 5.50 & $\checkmark .30$ & $\checkmark$ & 515.1477 & $191,179,135$ \\
3,5-diCQA (17) & 4.78 & $\checkmark$ & 515.1261 & $191,179,135$ \\
3-CQA glycoside (18) & 5.47 & $\checkmark$ & 515.1354 & $191,179,135$ \\
5-CQA glycoside (19) & & $\checkmark$ & $\checkmark$ & 191,135 \\
\hline
\end{tabular}

both leaf and cell suspension samples. Another interesting observation was the fact that cis-5-CQA (9) appeared in a relatively high intensity compared to the cis-3- CQA in both the leaf and cell samples. The same was also noted in our previous study where the same molecule was shown to be induced by activators of plant defence and priming responses [8]. The differences in the metabolite profiles (including CGAs and phenolic content) between cells and leaf tissue have been previously reported $[21,29]$. However, according to the best of our knowledge, this is the first report focusing on the differences between cells and leaf tissue samples taking into account both regional and geometrical isomerism (cis and trans configurations) of CGAs. From our previous work [8], we could confidently conclude that the cis-5-CQA molecule could be a natural product of tobacco plant systems, suggesting an interesting biochemical phenotype which is not fully explained in other plant species.

\section{Characterization of feruloylquinic acids}

Feruloylquinic acids have an $\mathrm{Mr}=368$. Similarly to $p$ CoQA and CQA, molecules harboring ferulic acid
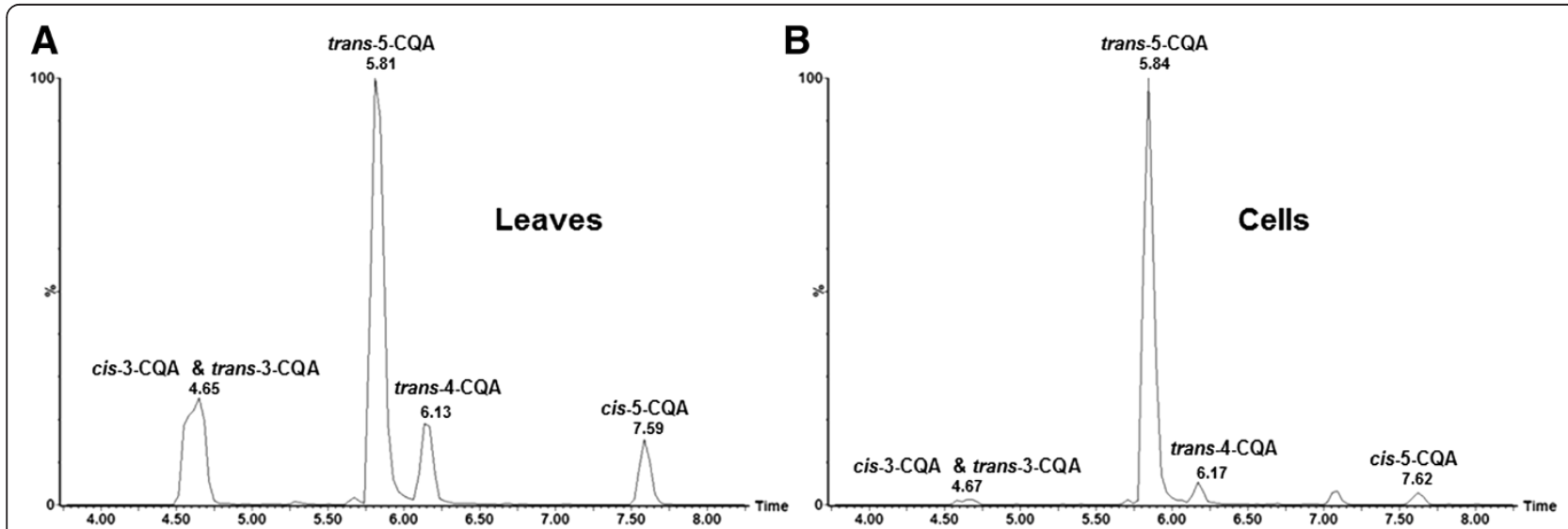

Figure 2 Representative extracted single ion chromatograms (XIC) of UPLC-MS/MS showing differential elution times of regio- and geometrical isomers of CQAs in methanol extracts of tobacco leaf tissue (A) and cultured cells (B). The cis-3-CQA and trans-3-CQA co-eluted at Rt $=4.6-4.7$ min. 

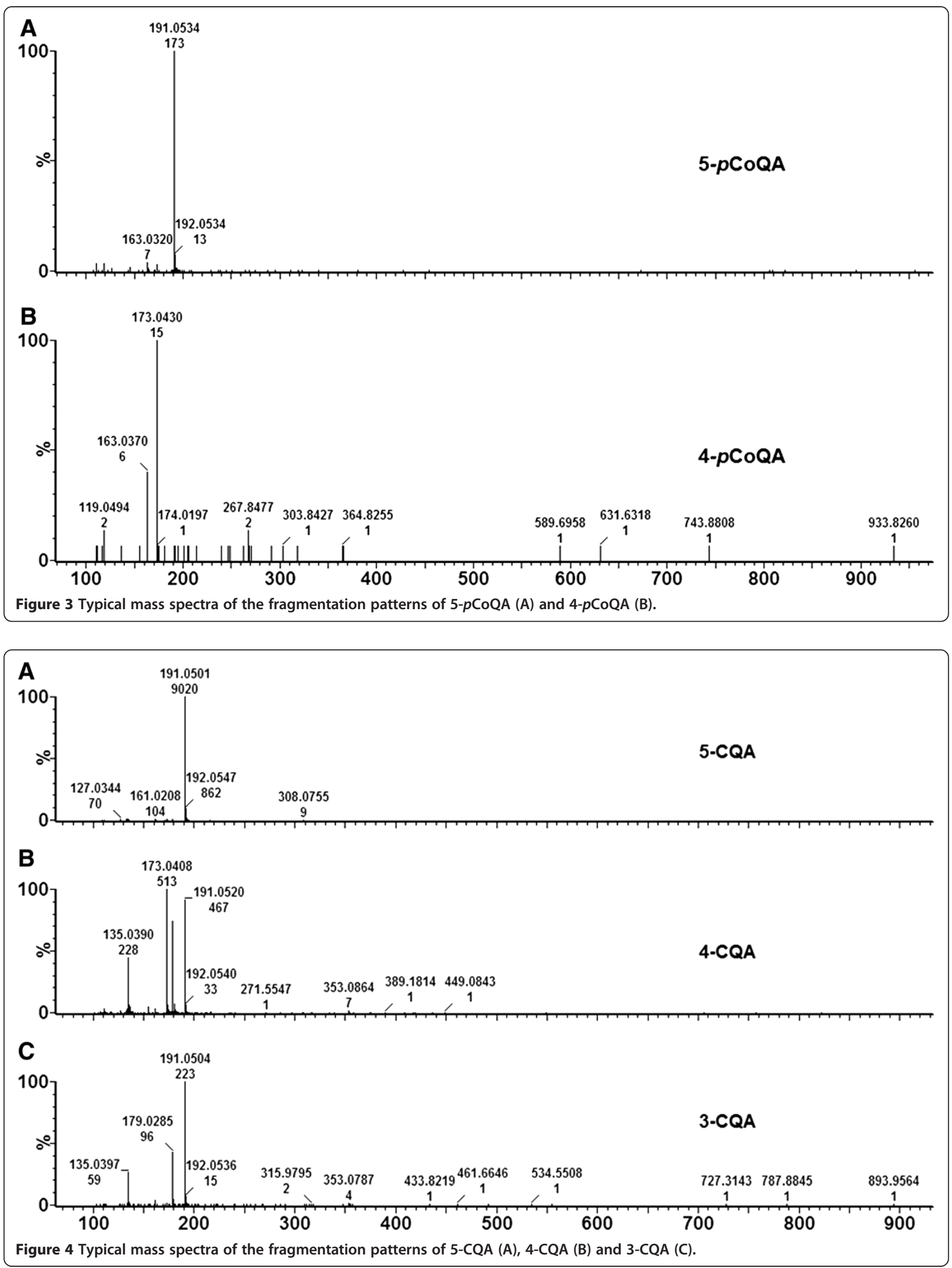

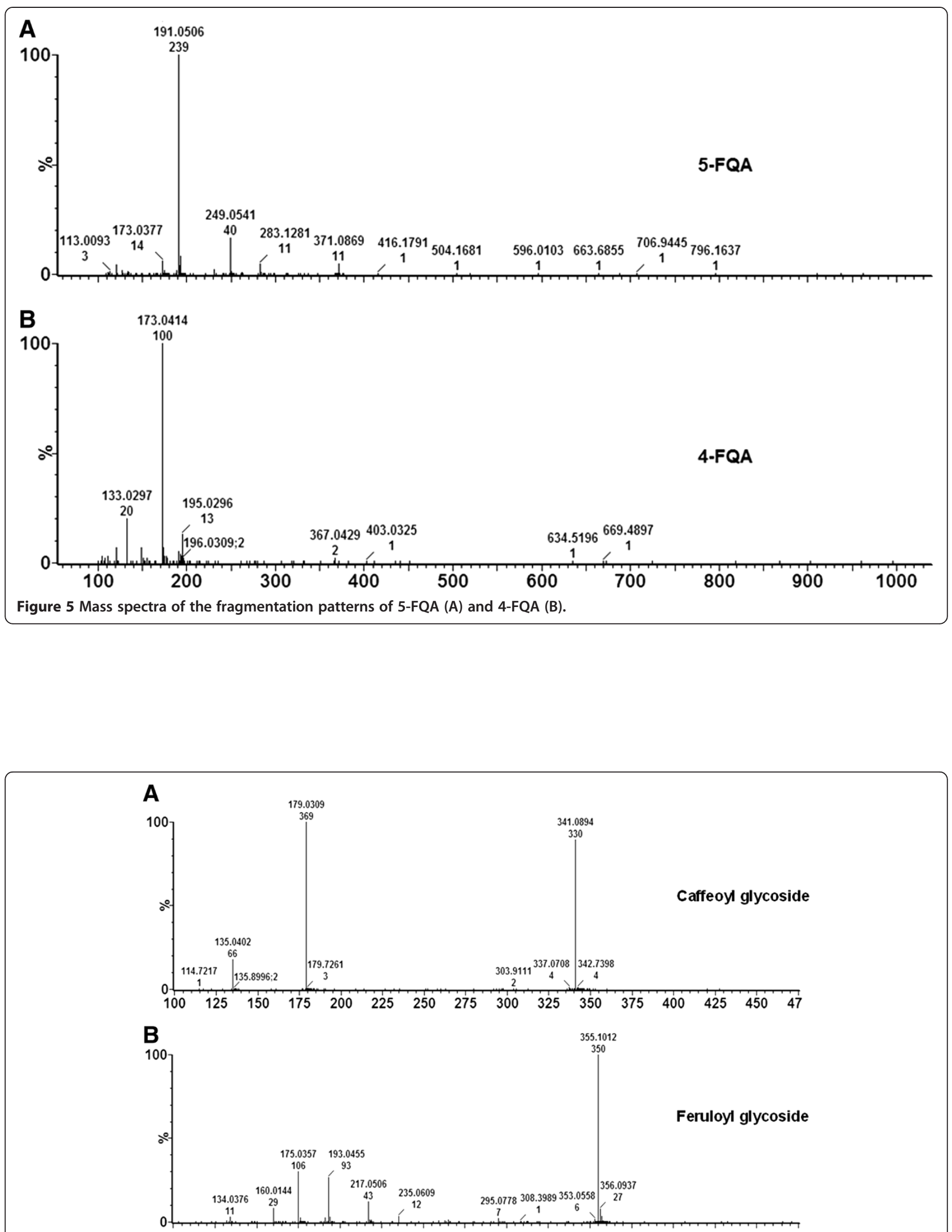

Figure 6 Mass spectra of the fragmentation patterns of caffeoylglycoside (A) and feruloylglycoside (B). 

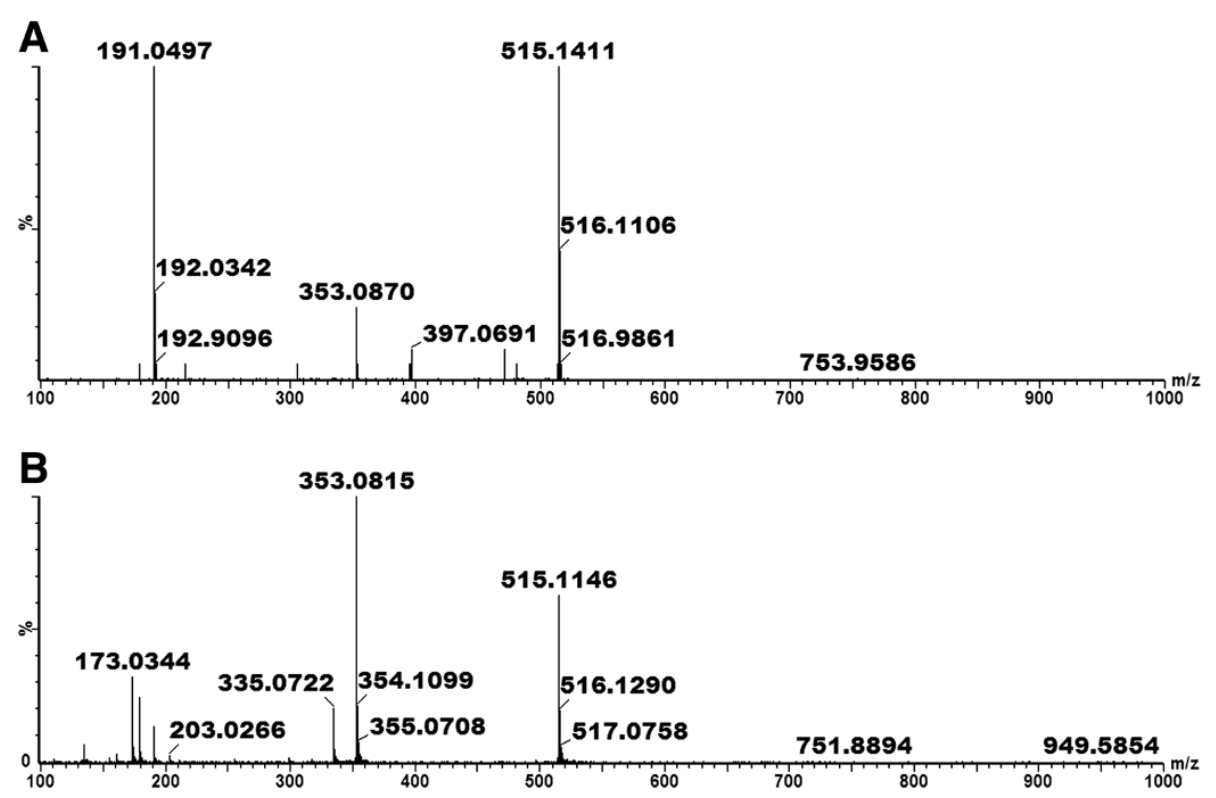

Figure 7 Mass spectra of the fragmentation patterns of 3,5-di-CQA (A), 4,5-di-CQA (B).

moieties were also identified (Figure 5). However, only three peaks were successfully identified; as trans-5-FQA (10), cis-5-FQA (11) and trans-4-FQA (12) respectively (Table 1). Similarly to the $p$ CoQA, there was an absence of the 3-acyl molecule in both cell- and leaf extracts. These three FQAs were identified in leaf tissue, however, only trans-5-FQA (10) and cis-5-FQA (11) were identified in cells, suggesting an underlying biochemical difference between the two systems, possibly due to enzymes differing in their substrate specificities.

\section{Characterization of caffeoylglycoside and feruloylglycoside}

Caffeoylglycoside and feruloylglycoside have molecular weights of 342 and 356 respectively. Two molecules with pseudomolecular peaks at $\mathrm{m} / z 341$ (Figure 6A) were identified as isomers of caffeoylglycoside (13) in both leaf and cell samples. They produced distinctive ions at $\mathrm{m} / z 179$ ([caffeic acid $-\mathrm{H}]-$ ) by the loss of a glucosyl residue $\left(\mathrm{C}_{6} \mathrm{H}_{10} \mathrm{O}_{5}\right)$ and $m / z 135$ ([caffeic acid - $\mathrm{H}$ ] -). As for the feruloylglycoside at $m / z 355$ (14, Figure 6B), it was also tentatively identified based on its fragmentation patterns: briefly this molecule produced a base peak at $\mathrm{m} / z 193$ ([ferulic acid - H]-) and by the loss of a glucosyl residue $\left(\mathrm{C}_{6} \mathrm{H}_{10} \mathrm{O}_{5}\right)(162 \mathrm{Da})$. It also produced a base peak at $m / z 175$ ([ferulic acid $\left.-\mathrm{H}-\mathrm{H}_{2} \mathrm{O}\right]-$ ), a peak at $m / z 295([\mathrm{M}-\mathrm{H}-60 \mathrm{Da}]-)$ by the loss of $\mathrm{C}_{2} \mathrm{H}_{4} \mathrm{O}_{2}$ and another peak at $m / z 235$ ([M-H $-120 \mathrm{Da}]-)$ by the loss of $\mathrm{C}_{4} \mathrm{H}_{8} \mathrm{O}_{4}$, due to internal sugar fragmentations. The identification of these molecules was also found to be consistent with published data [9]. Interestingly, this molecule was only identified in cells and not in leaf samples. The fact that feruloylglycoside molecules are only biosynthesized in cell suspensions again suggests a very interesting biochemical characteristic of the cultured cells in suspension which is absent in the leaf tissue.

\section{Characterization of di-caffeoylquinic acid and caffeoylquinic acid glucoside}

Both di-caffeoylquinic acid and caffeoylquinic acid glucoside have an $\mathrm{Mr}=516$. Here, a maximum of nine peaks at $m / z 515$ were identified and, based on the accurate masses and fragmentation patterns, these ions were distinguished as either di-caffeoylquinic acid and caffeoylquinic acid glycosides (Figure 7). As previously reported, both di-CQA and CQA-glycoside produce an isobaric pseudomolecular ion at $m / z 515$. As such, molecules 15-19 were annotated as either di-CQA or CQA glycosides. Based on the accurate mass, the diCQA were detected with an average $m / z$ of $515.1463\left(\mathrm{C}_{25} \mathrm{H}_{23} \mathrm{O}_{12}\right)$ and the CQA glycosides were found to have an average $m / z$ of $515.1292\left(\mathrm{C}_{22} \mathrm{H}_{27} \mathrm{O}_{14}\right)$. Based on information published elsewhere [10,11], molecules 15-17 were identified as di-CQAs (3,4 di-CQA (15), 4,5 di-CQA (16) and 3,5 di-CQA (17). Interestingly, both leaves and cells were found to contain these compounds except for the 3,4 di-CQA which was only present in the cells. Unlike the diCQA, the CQA glycosides produce distinctive ions at $m / z 341$ ([caffeoyl glucoside $-\mathrm{H}$ ]-) or/and 323 ([caffeoyl glucoside $\left.-\mathrm{H}-\mathrm{H}_{2} \mathrm{O}\right]-$ ) which were not present in the diCQA MS spectra. Recently, Jaiswal et al. $[30,31]$ reported the hierarchical fragmentation scheme of similar molecules, briefly it was noted that CQA 
forms a glycoside through an ether bond at either C-3 or $\mathrm{C}-4$ on the aromatic caffeoyl ring. During MS fragmentation, these molecules gives rise to ions at $\mathrm{m} / \mathrm{z} 341$ which predominates in both cases; however a peak at $\mathrm{m} / \mathrm{z}$ 323 is a characteristic of glucosyl attachment at C-3 [30]. Thus these molecules were putatively identified as 3-O(4'-O-caffeoyl glucosyl) quinic acid (18) since it also produced similar fragmentation to 3CQA. In turn, 5-O(3'-O-caffeoyl glucosyl) quinic acid (19) produces fragmentation similarly to 5-CQA (Figure 8). Interestingly, the CQA glycosides were only present in leaf tissue samples. Also, a previous report showed the accumulation of similar glycosides in Moringa leaves [32] and recently in Lonicera leaves [30].

\section{Conclusion}

Using the described UHPLC-Q-TOF-MS/MS fingerprinting method, based on the ISCID approach, a total of 19 different metabolites with a cinnamic acid core moiety were identified. Chlorogenic acids and related compounds are important because their involvement during plant defence responses is also becoming apparent $[8,33,34]$. In a separate study, the distribution of these molecules were found to be different across the different parts of the plants [35], which is an indication that there could be a localised function associated with a particular content and distribution. Similarly, the results of the current study indicate that there exists a significant difference in the CGA profiles of tobacco leaf tissue and cell suspensions. Possibly, the differences stem from different biochemical pathways leading to the biosynthesis of CGA molecules in the two biological systems. As already mentioned, the biochemical differences between cells and leaf tissue could be a result of the different environmental conditions which the cells and the leaves are exposed to as well as the level of tissue differentiation. This finding is in agreement with other published data where it was demonstrated that the CGA content varies with plant developmental stages. Therefore, there is a need to further investigate the underlying biochemical differences in such plant systems by investigating the same plant systems at transcriptomic and proteomic levels in conjunction with MS-based metabolite profiling.
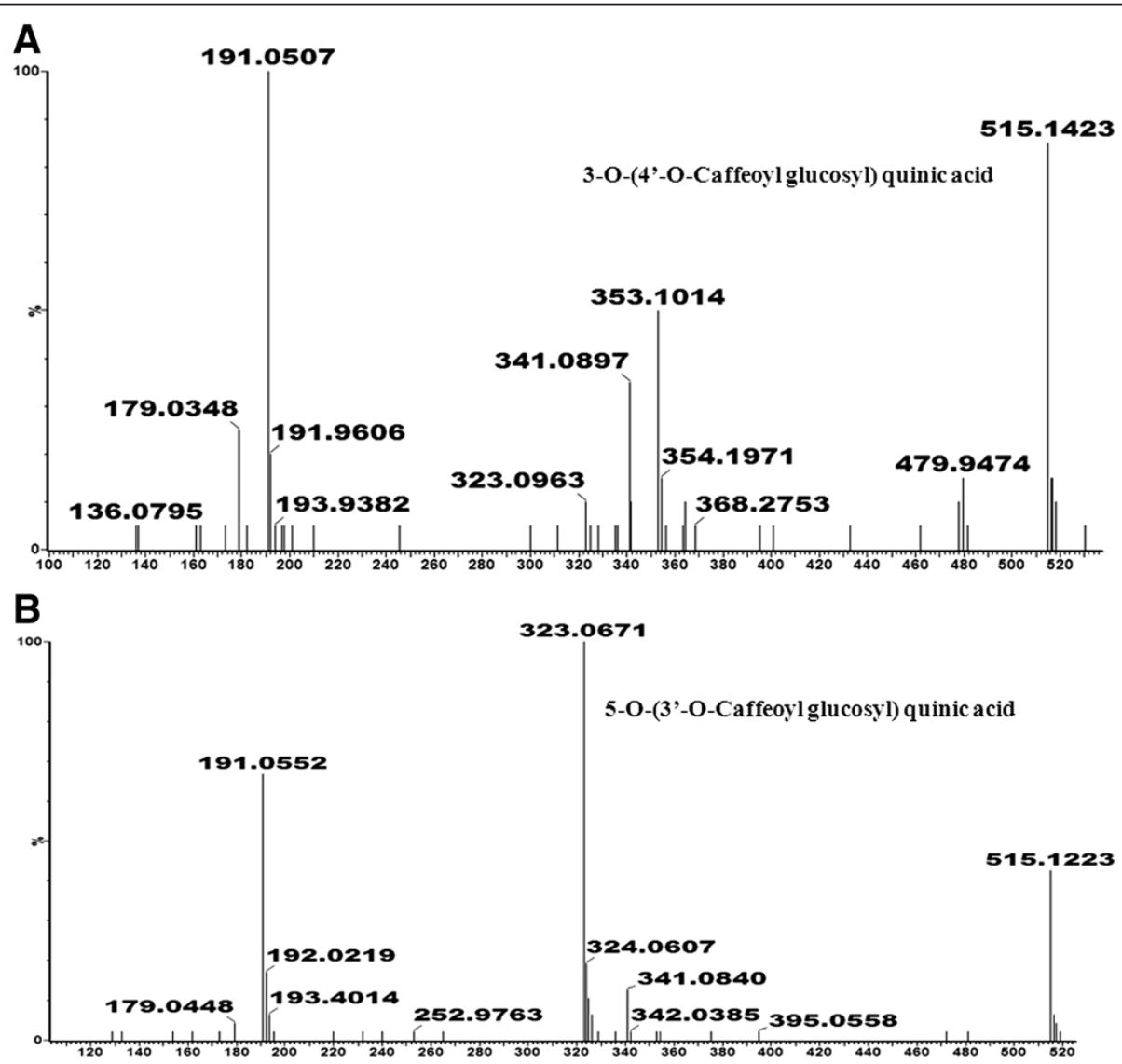

Figure 8 Mass spectra of the fragmentation patterns of 3-O-(4'-O-caffeoyl glucosyl) quinic acid (A) and 5-O-(3'-O-caffeoyl glucosyl) quinic acid (B). 


\section{Abbreviations}

CGA: Chlorogenic acids; CQA: Caffeoylquinic acid; UHPLC: Ultra-high performance liquid chromatography; ISCID: In-source collision-induced dissociation; Q-TOF-MS: Quadrupole time-of-flight mass spectrometry.

\section{Competing interests}

The authors declare that they have no competing interests.

\section{Authors' contributions}

NEM and IAD conceived of the study, ENN and MIM conducted the experiments and PAS and NEM the MS analyses. NEM and IAD supervised the project and LAP participated in its design and coordination and helped to draft the manuscript. All authors read and approved the final manuscript.

\section{Acknowledgements}

NEM and IAD received grants from the South African National Research Foundation (NRF). ENN and MIM were supported by NRF and University of Johannesburg fellowships. IAD and PAS received an equipment grant from the NRF's National Equipment Programme (NEP).

\section{Author details}

'Department of Biochemistry, University of Johannesburg, P.O. Box 524 Auckland Park 2006, South Africa. ${ }^{2}$ CSIR Biosciences, Natural Products and Agroprocessing Group, Pretoria 0001, South Africa.

Received: 28 August 2014 Accepted: 30 October 2014

Published online: 19 November 2014

\section{References}

1. Clifford MN: Chlorogenic acids and other cinnamates- nature, occurrence and dietary burden. J Sci Food Agr 1999, 79:362-372.

2. Clifford MN: Chlorogenic acids and other cinnamates nature, occurrence, dietary burden, absorption and metabolism. J Sci Food Agr 2000, 80:1033-1042.

3. Marques $V$, Farah A: Chlorogenic acids and related compounds in medicinal plants and infusions. Food Chem 2012, 113:1370-1376.

4. Koshiro Y, Jackson M-C, Katahiraa R, Wang M, Nagaib C, Ashiharaa H: Biosynthesis of chlorogenic acids in growing and ripening fruits of Coffea arabica and Coffea canephora plants. Z Naturforsch C 2007, 62:731-742.

5. Plazas M, Andújar I, Vilanova S, Hurtado M, Gramazio P, Herraiz FJ, Prohens J: Breeding for chlorogenic acid content in eggplant: interest and prospects. Bot Horti Agrobo 2013, 41:26-35.

6. Lou Z, Wang H, Zhu S, Ma C, Wang Z: Antibacterial activity and mechanism of action of chlorogenic acid. J Food Sci 2011, 76:398-403.

7. Xie C, Yu K, Zhong D, Yuan T, Ye F, Jarrell J-A, Millar A, Chen X: Investigation of isomeric transformations of chlorogenic acid in buffers and biological matrixes by Ultra Performance Liquid Chromatography coupled with hybrid quadrupole/ion mobility/orthogonal acceleration time-of-flight mass spectrometry. J Agr Food Chem 2011, 59:11078-11087.

8. Mhlongo MI, Piater LA, Steenkamp PA, Madala NE, Dubery IA: Metabolomic fingerprinting of primed tobacco cells provide the first evidence for the biological origin of cis-chlorogenic acid. Biotechnol Lett (in press)

9. Jaiswal R, Kuhnert N: Identification and characterization of the phenolic glycosides of Lagenaria siceraria Stand (Bottle Gourd) fruit by liquid chromatography-tandem mass spectrometry. J Agr Food Chem 2014, 62:1261-1271.

10. Clifford M-N, Johnston K-L, Knight S, Kuhnert N: A hierarchical scheme for LCMS $^{\mathrm{n}}$ identification of chlorogenic acid. J Agr Food Chem 2003, 53:3821-3832.

11. Clifford MN, Knight S, Kuhnert N: Discriminating between the six isomers of dicaffeoylquinic acid by LC-MS ${ }^{n}$. J Agr Food Chem 2005, 53:3821-3832.

12. Clifford M-N, Kirkpatrick J, Kuhnert N, Roozendaal H, Salgado PR: LC-MS ${ }^{n}$ analysis of the cis isomers of chlorogenic acids. Food Chem 2008, 106:379-385.

13. Kuhnert N, Karaköse $H$, Jaiswal R: Analysis of chlorogenic acids and other hydroxycinnamates in food, plants and pharmacokinetic studies. In Handbook of Analysis of Active Compounds in Functional Foods, Volume 1. Edited by Nollet ML L, Toldra F. United States: CRC Press; 2012:461.

14. Wang J, Lu D, Zhao $H$, Jiang $B$, Wang J, Ling $X$, Chai $H$, Ouyang $P$. Discrimination and classification of tobacco wastes by identification and quantification of polyphenols with LC-MS/MS. J Serb Chem Soc 2010, 75:875-891.

15. Ramachandra RS, Ravishankar GA: Plant cell cultures: Chemical factories of secondary metabolites. Biotechnol Adv 2002, 20:101-153.
16. Verpoorte R, Contin A, Memelink J: Biotechnology for the production of plant secondary metabolites. Phytochem Rev 2002, 1:13-25.

17. Kolewe ME, Gaurav V, Roberts SC: Pharmaceutically active natural product synthesis and supply via plant cell culture technology. Mol Pharm 2008, 5:243-256

18. Atkinson MM, Huang J, Knopp JA: Hypersensitivity of suspension-cultured tobacco cells to pathogenic bacteria. Phytopathology 1985, 75:1270-1274.

19. Mozzetti C, Ferraris L, Tamietti G, Matta A: Variation in enzyme activities in leaves and cell suspensions as markers of incompatibility in different Phytophthora pepper interactions. Physiol Mol Plant P 1995, 46:95-107.

20. Franklin G, Dias ACP: Chlorogenic acid participates in the regulation of shoot, root and root hair development in Hypericum perforatum. Plant Physiol Biochem 2011, 49:835-842.

21. Mondolot L, La Fisca P, Buatois B, Talansier E, De Kochko A, Campa C: Evolution in caffeoylquinic acid content and histolocalization during Coffea canephora leaf development. Ann Bot 2006, 98:33-40.

22. Sanabria NM, Dubery IA: Differential display profiling of the Nicotiana response to LPS reveals elements of plant basal resistance. Biochem Biophys Res Commun 2006, 344:1001-1007.

23. Hossain MB, Rai D-K, Brunton NP, Martin-Diana AB, Barry-Ryan C: Characterization of phenolics composition in Lamiaceae spices by LCESI-MS/MS. J Agr Food Chem 2010, 58:10576-10581.

24. He W, Liu X, Xu H, Gong Y, Yuan F, Gao Y: On-line HPLC-ABTS screening and HPLC-DAD-MS/MS identification of free radical scavengers in Gardenia (Gardenia jasminoides Ellis) fruit extracts. Food Chem 2010, 123:521-528.

25. Nurmi K, Ossipov V, Haukioja E, Pihlaja K: Variation of total phenolic content and individual low-molecular-weight phenolics in foliage of mountain birch trees (Betula pubescens ssp. tortuosa). J Chem Ecol 1996, 22:2023-2040.

26. Riipi M, Ossipov V, Lempa K, Haukioja E, Koricheva J, Ossipova S, Pihlaja K: Seasonal changes in birch leaf chemistry: are there trade-offs between leaf growth and accumulation of phenolics. Oecologia 2002, 130:380-390.

27. Regos I, Urbanella A, Treutter D: Identification and quantification of phenolic compounds from the forage legume sainfoin (Onobrychis viciifolia). J Agr Food Chem 2009, 57:5843-5852.

28. Farah A, Monteiro M, Donangelo CM, Lafey S: Chlorogenic acids from green coffee extracts are highly bioavailable in humans. J Nutr 2008, 138:2309-2315.

29. De Mello M-O, De Campos Amaral AF, Melo M: Sucrose metabolizing enzymes in cell suspension cultures of Bauhinia forficata, Curcuma zedoaria and Phaseolus vulgaris. Pesqui Agropecu Bras 2001, 36:1085-1092.

30. Jaiswal R, Muller H, Muller A, Karar MGE, Kuhnert N: Identification and characterisation of chlorogenic acids, chlorogenic acid glycosides and flavonoids from Lonicera henryi L. (Caprifoliaceae) leaves by LC-MS ${ }^{n}$. Phytochemistry 2014, 10.1016/j.phytochem.2014.08.023

31. Jaiswal R, Halabi EA, Karar MGE, Kuhnert N: Identification and characterisation of the phenolics of Ilex glabra L. Gray (Aquifoliaceae) leaves by liquid chromatography tandem mass spectrometry. Phytochemistry 2014, 106:141-155.

32. Kashiwada Y, Ahmed FA, Kurimoto S, Kim SY, Shibata H, Fujikota T, Takaishi $Y$ : New alpha-glucosides of caffeoyl quinic acid from the leaves of Moringa oleifera Lam. J Nat Med 2012, 66:217-221.

33. Mhlongo MI, Piater LA, Steenkamp PA, Madala NE, Dubery IA: Priming agents of plant defence stimulate the accumulation of mono- and $\mathrm{di}$ acylated chlorogenic acids in cultured tobacco cells. Physiol Molec Plant Pathol 2014 (in press)

34. Torras-Claveria L, Jáuregui O, Codinaa C, Tiburcioa AF, Bastidaa J, Viladomat F: Analysis of phenolic compounds by high-performance liquid chromatography coupled to electrospray ionization tandem mass spectrometry in senescent and water-stressed tobacco. Plant Sci 2012, 182:71-78.

35. Parveen I, Threadgill MD, Hauck B, Donnison I, Winters A: Isolation, identification and quantitation of hydroxycinnamic acid conjugates, potential platform chemicals, in the leaves and stems of Miscanthus $x$ giganteus using LC-ESI-MS(n). Phytochemistry 2012, 72:71-78.

doi:10.1186/s13065-014-0066-z

Cite this article as: Ncube et al:: Analyses of chlorogenic acids and related cinnamic acid derivatives from Nicotiana tabacum tissues with the aid of UPLC-QTOF-MS/MS based on the in-source collision-induced dissociation method. Chemistry Central Journal 2014 8:66. 